\title{
Selenolysine: A New Tool for Traceless Isopeptide Bond Formation
}

Rebecca Notis Dardashti, Shailesh Kumar, Shawn M. Sternisha, Post Sai Reddy, Brian G. Miller, Norman Metanis

Submitted date: 19/12/2019 - Posted date: 24/12/2019

Licence: CC BY-NC-ND 4.0

Citation information: Dardashti, Rebecca Notis; Kumar, Shailesh; Sternisha, Shawn M.; Reddy, Post Sai;

Miller, Brian G.; Metanis, Norman (2019): Selenolysine: A New Tool for Traceless Isopeptide Bond Formation. ChemRxiv. Preprint. https://doi.org/10.26434/chemrxiv.11406546.v1

Despite their biological importance, post-translationally modified proteins are notoriously difficult to produce in a homogeneous fashion using conventional expression systems. Chemical protein synthesis or semi-synthesis offers a solution to this problem; however, traditional strategies often rely on sulfur-based chemistry that is incompatible with the presence of multiple cysteine residues in the target protein. To overcome these limitations, we present the design and synthesis of $\gamma$-selenolysine, a selenol-containing form of the commonly modified proteinogenic amino acid, lysine. The utility of $\gamma$-selenolysine is demonstrated with the traceless ligation of the small ubiquitin-like modifier protein, SUMO-1, to a peptide segment of human glucokinase. The resulting polypeptide is poised for native chemical ligation and selective deselenization in the presence of unprotected cysteine residues. Selenolysine's straightforward synthesis and incorporation into synthetic peptides marks it as a universal handle for conjugating any ubiquitin-like modifying protein to its target.

File list (1) 


\title{
Selenolysine: a new tool for traceless isopeptide bond formation
}

\author{
Rebecca Notis Dardashti ${ }^{1}$, Shailesh Kumar ${ }^{1}$, Shawn M. Sternisha ${ }^{2}$, Post Sai Reddy ${ }^{1}$, \\ Brian G. Miller ${ }^{2}$, and Norman Metanis ${ }^{1, *}$ \\ ${ }^{1}$ The Institute of Chemistry, The Hebrew University of Jerusalem, Edmond J. Safra \\ Campus, Givat Ram, Jerusalem 91904, Israel \\ ${ }^{2}$ Department of Chemistry and Biochemistry, Florida State University, Tallahassee, \\ FL 32306-4390 \\ Correspondence should be addressed to: Metanis@mail.huji.ac.il
}

\begin{abstract}
Despite their biological importance, post-translationally modified proteins are notoriously difficult to produce in a homogeneous fashion using conventional expression systems. Chemical protein synthesis or semi-synthesis offers a solution to this problem; however, traditional strategies often rely on sulfur-based chemistry that is incompatible with the presence of multiple cysteine residues in the target protein. To overcome these limitations, we present the design and synthesis of $\gamma$-selenolysine, a selenol-containing form of the commonly modified proteinogenic amino acid, lysine. The utility of $\gamma^{-}$ selenolysine is demonstrated with the traceless ligation of the small ubiquitin-like modifier protein, SUMO-1, to a peptide segment of human glucokinase. The resulting polypeptide is poised for native chemical ligation and selective deselenization in the presence of unprotected cysteine residues. Selenolysine's straightforward synthesis and incorporation into synthetic peptides marks it as a universal handle for conjugating any ubiquitin-like modifying protein to its target.
\end{abstract}




\section{Introduction}

Following ribosomal translation and folding, expressed proteins can undergo a great number of post-translational modifications (PTMs) before fulfilling their biological functions. ${ }^{1}$ These PTMs instruct the cell to carry out a variety of regulatory activities including transportation, degradation, and others. ${ }^{2}$ Included in these covalent modifications are well-known phosphorylation and glycosylation of nucleophilic amino acids. Additionally, Lys, which is one of the more reactive amino acids under biological conditions, can be modified with post-translationally modifying proteins such as ubiquitin and other ubiquitin-like modifiers (Ubl). ${ }^{3}$

Techniques for controlling PTMs in cellular expression are not yet sufficiently welldeveloped to allow recombinant expression of homogenously modified proteins. ${ }^{4}$ Alternatively, some methods for semi-selective modification of expressed proteins have been developed, most employing Cys chemistry. For example, dehydroalanine (Dha), a suitable electrophile for Michael addition reactions, can be generated from Cys, ${ }^{5}$ Ser, ${ }^{6}$ thioethers, ${ }^{7,8}$ and several selenium-containing unnatural amino acids. ${ }^{9}$ Michael addition to the resulting Dha has been used to introduce many small-molecule PTMs and entire PTM proteins, as in the case of ubiquitinated $\alpha$-globin. ${ }^{10}$ However, a stereoselective method for addition to Dha has yet to be developed, ${ }^{10}$ a drawback in the chiral world of biology.

Chemical protein synthesis (CPS) and semi-synthesis, on the other hand, offers a direct route to selectively modified proteins. ${ }^{11}$ Based on solid-phase peptide synthesis (SPPS) ${ }^{12}$ and native chemical ligation (NCL) ${ }^{13}$, this approach allows the preparation of modified proteins, including proteins with multiple sites of different PTMs. ${ }^{4}$

Additionally, Lys-mediated chemical ligation to form an isopeptide bond, typically referred to as isopeptide chemical ligation (ICL), has been used as a tool to conjugate post-translationally modifying proteins to their targets. Previously, Muir and coworkers reported ubiquitinylated protein synthesis using a modified Lys with an auxiliary that was removed post-ligation. ${ }^{14,15}$ Brik and coworkers introduced $\delta$-thiolysine in the conjugation of ubiquitin and synthesis of ubiquitinated proteins or ubiquitin chains. ${ }^{16-18}$ Another Lys derivative, $\gamma$-thiolysine, has been used as a handle in dual NCL reactions, ${ }^{19,20}$ in which the $\mathrm{\gamma}$-thiolysine plays a role in both main chain and isopeptide ligation. Global desulfurization of the final products gives a native Lys at the ligation site (Scheme 1). 


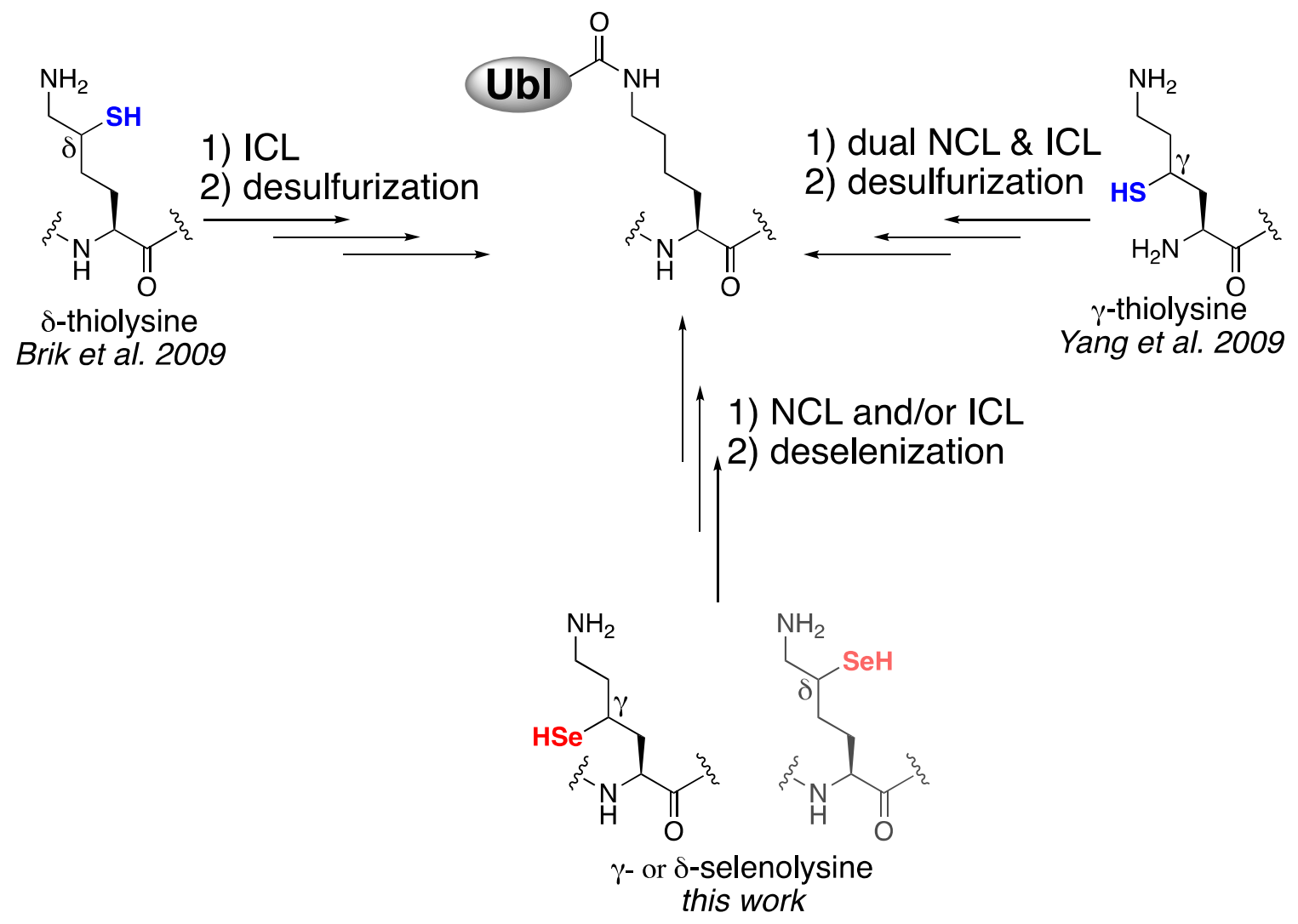

Scheme 1. Previously developed $\delta$-thiolysine, by Brik ${ }^{16,17}$, and $\gamma$-thiolysine, by Yang ${ }^{19,20}$, used for NCL and ICL followed by desulfurization for the conjugation of Ubl proteins to Lys residues. Our designed $\gamma / \delta$-selenolysines for NCL and/or ICL followed by chemoselective deselenization for traceless conjugation of Ubl proteins to Lys residues.

We sought to develop selenium-containing analogs of modified Lys residues to facilitate traceless isopeptide bond formation through ICL and a subsequent, chemoselective deselenization reaction in the presence of unprotected Cys residues (Scheme 1). The aforementioned $\gamma / \delta$-thiolysines used in ICL/desulfurization approaches are not compatible in the presence of proteins with natural Cys residues in their sequences, unless orthogonal Cys protecting groups are installed. This presents a true challenge in EPL, where installation of protected Cys residues in Ubl proteins would require specialized expression methods. In contrast, thanks to selenium's unique chemical properties, $\gamma / \delta$-selenolysine (Se-Lys, Sek, or $\mathrm{K}^{*}$ ) could be used in an ICL/deselenization approach in the presence of unprotected Cys residues, without the risk of desulfurizing Cys residues. ${ }^{21,22}$ 
Here we report the synthesis and use of $\gamma$-Se-Lys as a new tool for traceless isopeptide bond formation. The design and synthesis of $\delta$-Se-Lys, which could not be completed due to spontaneous Se-elimination, is described in the SI (Scheme S1).

\section{Results and discussion}

To obtain a protected form of $\gamma$-Se-Lys suitable for SPPS, we turned to work by Merkx et al., where an Fmoc-Lys[S $\left.\mathrm{S}^{\gamma}(\mathrm{StBu})-\mathrm{N}^{\varepsilon}(\mathrm{Boc})\right]-\mathrm{OH}$ was successfully synthesized from $\gamma^{-}$ chlorolysine. ${ }^{23}$ We opted to follow the previous protocol until selenium incorporation, protecting the $\alpha$-amino and carboxylate groups of $\gamma$-chlorolysine with 9-BBN and then the $\varepsilon-\mathrm{NH}_{2}$ group with Boc to give 2 (Scheme 2).

Scheme 1. Synthesis of Fmoc-Lys[Se $\left.\mathrm{Se}^{\gamma}(\mathrm{Mob})-\mathrm{N}^{\varepsilon}(\mathrm{Boc})\right]-\mathrm{OH}$

Nucleophilic substitution of $\mathrm{Cl}$ to Se in DMF was performed using $\mathrm{Na}_{2} \mathrm{Se}_{2}$, generated in situ from Se powder, hydrazine monohydrate, and $\mathrm{NaOH}^{24}$ The reaction gave the $\mathrm{\gamma}$ diselenide product 3 in $42 \%$ yield, the maximum yield that could be obtained with a variety of different approaches and reagents. A reduction with $\mathrm{NaBH}_{4}$ and protection with p-methoxybenzyl chloride (PMB-Cl or Mob-Cl) gave 4 in $60 \%$ yield. Finally, removal of 9-BBN and subsequent Fmoc-protection gave the desired Fmoc-Lys[Se $\left.{ }^{\gamma}(\mathrm{Mob})-\mathrm{N}^{\varepsilon}(\mathrm{Boc})\right]-$ $\mathrm{OH}, \mathbf{6}$, in 77\% yield (Scheme 2).

Following our successful synthesis of a protected Se-Lys building block for SPPS, we set out to design an analog with an orthogonal protecting group at the $\varepsilon-\mathrm{NH}_{2}$ site to allow for dual chemical ligation. In the proposed application, Se-Lys with an orthogonal protection at $\varepsilon-\mathrm{NH}_{2}$ would first be used in NCL along the main peptide chain. After NCL, the 
protecting group of $\varepsilon-\mathrm{NH}_{2}$ would be removed to allow for a second ligation, forming an isopeptide bond with the desired PTM protein-thioester. Final deselenization would give a native Lys at the PTM site (Scheme 3).

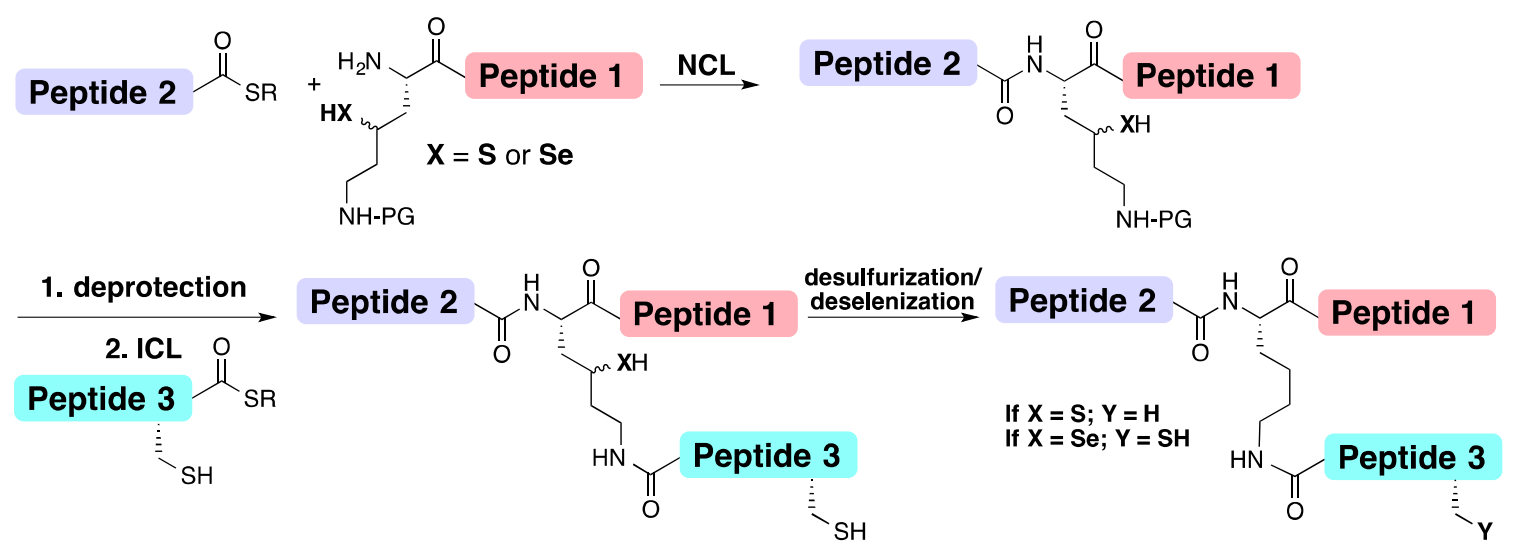

Scheme 3. $\gamma$-thiolysine ${ }^{19,20}$ and $\gamma$-selenolysine as tools for dual NCL and ICL at Lys. Selenolysine has the advantage of allowing chemoselective deselenization in the presence of unprotected Cys residues.

Disappointingly, using $\gamma$-chlorolysine, we were unsuccessful in incorporating an orthogonal protecting group on the $\varepsilon-\mathrm{NH}_{2}$. Instead, an alternative route was pursued from known aldehyde 10, which can be obtained from $L$-aspartic acid precursor in 3 steps (Scheme 4) ${ }^{25}$ In this synthesis (Scheme 4), the aldehyde 10 was treated with methyl (triphenylphosphoranylidene)acetate to afford the $\alpha, \beta$-unsaturated ester $\mathbf{1 1}$ (85\% for 2 steps). ${ }^{26}$ Michael addition on $\mathbf{1 1}$ with Mob-Selenolate (generated in situ from (Mob-Se-) 2 and $\left.\mathrm{NaBH}_{4}\right)^{27}$ gave compound $\mathbf{1 2}$ in $90 \%$ yield. The less sterically hindered ester in $\mathbf{1 2}$ was selectively converted to an aldehyde with DIBAL-H in ether (dried in $4 \AA$ MS) at -78 ${ }^{\circ} \mathrm{C}$, which was treated with $\mathrm{NaBH}_{4}$ in EtOH to give alcohol 13 (68\% for 2 steps). The hydroxyl group in $\mathbf{1 3}$ was converted to mesylate using $\mathrm{MsCl}$ and $\mathrm{Et}_{3} \mathrm{~N}$ in $\mathrm{CH}_{2} \mathrm{Cl}_{2}$ followed by treatment with $\mathrm{NaN}_{3}$ in DMF at $80{ }^{\circ} \mathrm{C}$, which afforded the azide compound 14 in 88\% yield. Reduction of azide 14 with $\mathrm{Ph}_{3} \mathrm{P}$ in $\mathrm{MeOH}$, followed by treatment with Alloc-Cl and $\mathrm{Et}_{3} \mathrm{~N}$, gave an Alloc-protected amine 15 (71\%).

Conversion of di-Boc (15) to mono-Boc (16) with $\mathrm{LiBr}^{28}$ was carried out in $\mathrm{CH}_{3} \mathrm{CN}$ at 65 ${ }^{\circ} \mathrm{C}$ for $24 \mathrm{~h}$ in $98 \%$ yield. Finally, hydrolysis of methyl ester with aqueous $\mathrm{LiOH}^{16}$ in aqueous THF gave the carboxylic acid $\mathbf{1 7}$ in 97\% yield (17 can be used in Boc-SPPS). Compound 17 was treated with TFA in $\mathrm{CH}_{2} \mathrm{Cl}_{2}$ to give an amine-salt, which was finally 
converted to Fmoc-protected amino acid 18 using Fmoc-OSu and aqueous $\mathrm{Na}_{2} \mathrm{CO}_{3}$ in $90 \%$ yield.

The final product, Fmoc-Lys[Se ${ }^{\gamma}(\mathrm{Mob})-\mathrm{N}^{\varepsilon}($ Alloc $\left.)\right]-\mathrm{OH}$, was achieved in $17 \%$ overall yield for 13 steps of synthesis (Scheme 4). Following successful main-chain NCL, the Alloc protecting group can be removed off-resin with a ruthenium catalyst ${ }^{29}$ and the resulting peptide can be subjected to isopeptide ligation. Studies showing the utility of Fmoc-Lys[N ${ }^{\varepsilon}\left(\right.$ Alloc)-Se $\left.{ }^{\gamma}(\mathrm{Mob})\right]-\mathrm{OH}$ in dual chemical ligation are currently underway.

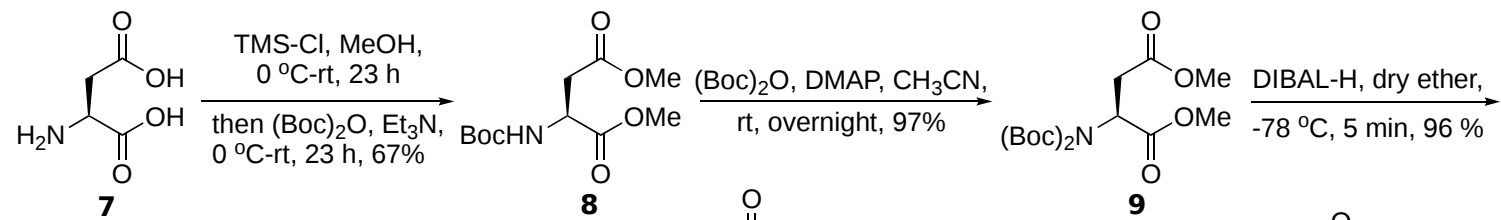

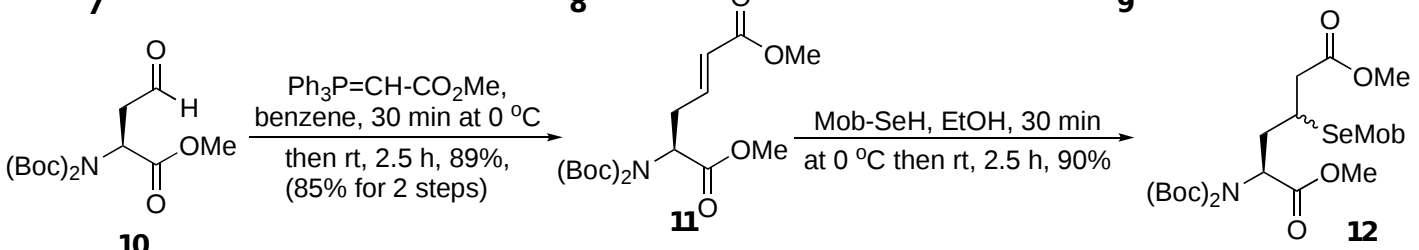

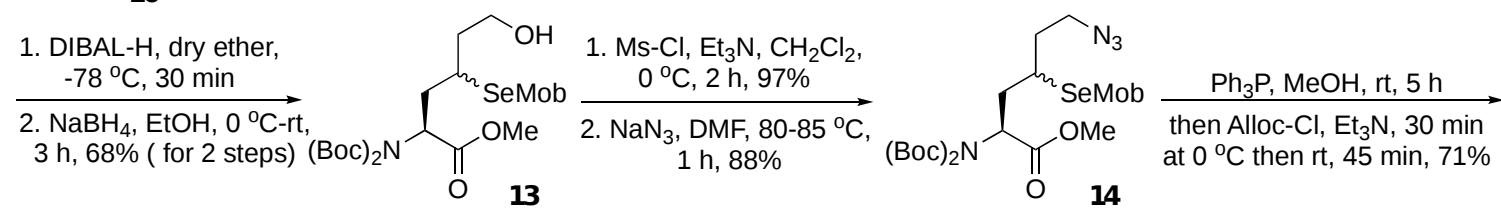

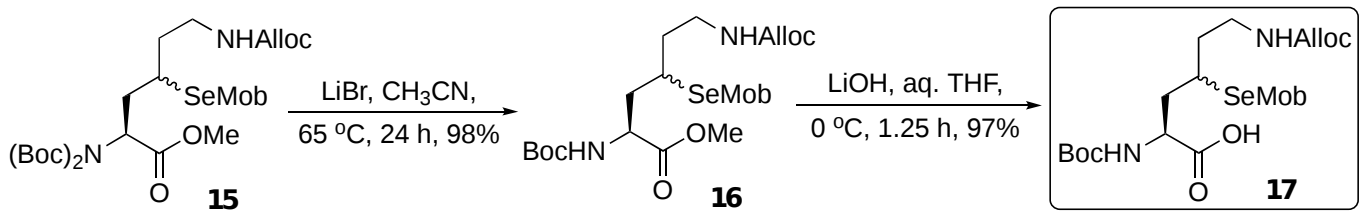

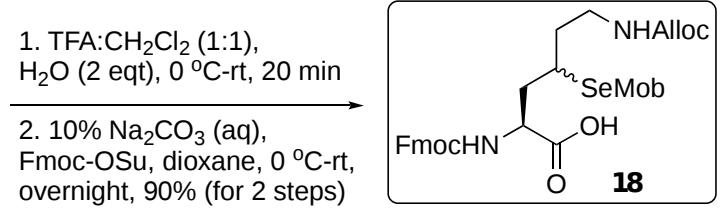


Scheme 4. Synthesis of protected form of $\mathrm{\gamma}$-Se-Lys for Boc- and Fmoc-SPPS.

To assess the utility of $\gamma$-Se-Lys in protein semi-synthesis, we incorporated our firstgeneration Se-Lys 6 (Scheme 2) into post-translationally modified human glucokinase (GCK). Prior studies demonstrated that the pancreatic isoform of this key glucose homeostatic enzyme is modified by the small ubiquitin-like modifier protein, SUMO-1, at one or more lysine residues near the N-terminus. ${ }^{30,31}$ Unfortunately, the structural and functional impact of site-specific GCK SUMOylation has been difficult to assess due to an inability to produce homogenously SUMOylated GCK. We viewed the SUMO-GCK pair as an attractive target, since both proteins contain unprotected cysteine residues, and thus require the unique chemical properties of $\gamma$-Se-Lys.

We devised a three-segment strategy to obtain semisynthetic SUMOylated human GCK, in order to evaluate the impact of this PTM on enzyme function (Scheme 5). First, SPPS was used to incorporate our Fmoc-protected $\gamma$-Se-Lys 6 at residue $15^{* 32-36}$ within a peptide consisting of the first 19 residues of GCK, GCK(2-19)- $\mathrm{NHNH}_{2}$ (Scheme 5, Figure S1). This peptide also contained a C-terminal masked thioester for downstream use in NCL ${ }^{37-39}$. Next, SUMO-1 was produced as a C-terminal fusion with the MxeGyrA C-terminal intein (Figures S2 and S3), ${ }^{40}$ which was subsequently converted to its 2mercaptoethane sulfonic acid thioester (SUMO-1-Mes) for ICL (Figure S4, details and method in SI). Following ICL, the product underwent a one-pot deselenization/thioesterification (Figure S5), giving an overall yield of $12.3 \%$ of the GCK(2-19) segment linked to SUMO-1 via an isopeptide bond at Lys15 (Figure 1).

\footnotetext{
F $^{*}$ Using online analysis programs such as SUMOplot ${ }^{\mathrm{TM}}$ or SUMOsp 2.0, which predict possible SUMOylation sites, K12 and K15 are predicted as likely SUMOylation sites in the N-terminus of pancreatic GCK. [31-32] The first site, K12, is part of SUMO consensus motif $\psi-\mathrm{K}-\mathrm{x}-\mathrm{D} / \mathrm{E}$, where $\psi$ is a large hydrophobic amino acid, $\mathrm{K}$ is the lysine at which the SUMO is linked, $\mathrm{x}$ is any amino acid and $\mathrm{D} / \mathrm{E}$ is an acidic amino acid (Asp or Glu, respectively).[34] The second SUMOylation site is K15, which is not part of a typical SUMO consensus motif. Both analysis programs did not predict K13 as a possible SUMOylation site. In early crystal structures of GCK (PDB entries 1V4S, 1V4T)[31] the N-terminus was truncated to facilitate crystallization. In these structures the N-terminal region of GCK, which begins with Lys12, adopts an $\alpha$-helix.[35] Although K12, K13 and K15 are adjacent residues, each residue would face a different environment as part of the $\alpha$-helix, with some inaccessible to the SUMOylation system. Indeed, based on a more recent crystal structure (PDB entry 3IDH),[35] only K15 is solvent exposed, while K12 and $\mathrm{K} 13$ are on the opposite face of the helix and make electrostatic and hydrogen bonding interactions with the rest of the protein. Based on these observations, we initially focused on Lys15 as the SUMOlylation site in pancreatic GCK; however, our method should be applicable to Lys12 or any Lys residue in protein sequence.
} 


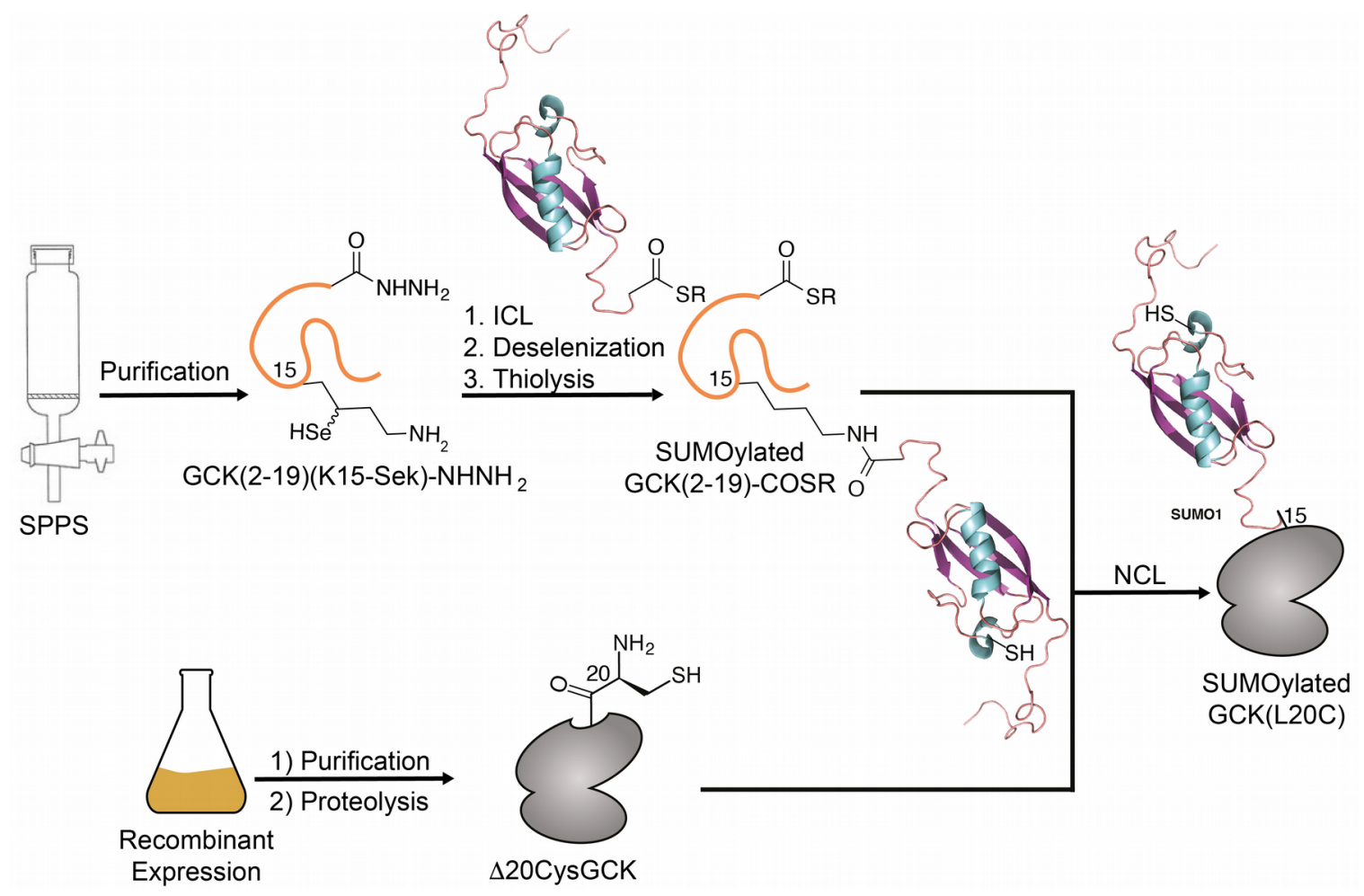

Scheme 5. Proposed synthesis of the semisynthetic SUMOylated GCK.

We expected the ligation step to proceed efficiently, as the size of the amino acid adjacent to the thioester typically dictates the rate of the ligation reaction. ${ }^{41}$ SUMO-1 contains a desirable Gly-Gly sequence adjacent to the thioester. However, the ligation step was surprisingly slow. SUMO-1-Mes was converted to the more reactive SUMO-1MPAA thioester within 1 hour. Yet, ligation of this adduct with the small GCK peptide took up to 22 hours to complete despite a large excess of the peptide and the presence of TCEP and sodium ascorbate, which are known to accelerate the selenium-mediated NCL reaction. ${ }^{22,} 42,43$ It is unlikely that the $\mathrm{Y}$-position of the selenol during ligation contributes to a slower rate of ligation, based on a previous report indicating that this has no impact of reaction rate. ${ }^{44}$ Instead, a direct comparison of isopeptide ligation in the synthesis of diubiquitin with $\gamma$ - and $\delta$-thiolysine also showed a slower-than-expected rate of ligation. ${ }^{23}$ Together, these observations suggest that the problem lies in the nature of isopeptide ligation, rather than the placement of thiol or selenol on the Lys side chain.

Due to the slow rate of ligation, a significant portion of SUMO-1-MPAA underwent hydrolysis to inactive SUMO-1-OH rather than to the desired ligation. As a result, the 
maximum yield we observed was $22 \%$ for the ligation step, which severely limited the expected yield of the overall strategy.

Notably, for the subsequent deselenization reaction, the unprotected, native Cys52 of SUMO-1 was unaffected by this procedure ${ }^{45}$ and unmasking of the peptide's cryptic thioester provided a segment poised for NCL.

As a final step in the preparation of SUMOylated human GCK, we recombinantly produced a human GCK variant containing a Factor Xa recognition sequence directly upstream of a Cys at position 20 (Scheme 5, and Figure S6). Following purification and factor Xa digestion, this procedure yielded a truncated, N-terminal Cys containing GCK variant, $\Delta$ 20CysGCK, that displayed wild-type kinetic characteristics (Figure S7). Unfortunately, efforts to perform native chemical ligation between $\Delta 20$ CysGCK and the SUMO-1-GCK(1-19)-COSR fragment proved unsuccessful. We observed no ligation after prolonged incubation of the reaction components in the absence of denaturants, whereas inclusion of guanidium hydrochloride in the ligation buffer caused extensive precipitation of the protein. ${ }^{46}$ Ultimately, the limited quantities of the synthetic SUMO-1GCK(1-19)-MPAA segment prevented us from exploring a variety of reaction conditions and additives to optimize ligation efficiency. Future efforts will focus on overcoming the limitations of the isopeptide ligation process to alleviate this issue. 



Figure 1. A. HPLC traces of one-pot SUMO-1-GCK deselenization and thioesterification over time. 1 is SUMO-1-GCK(2-19)- $\mathrm{NHNH}_{2}$ (K15Sek), 2 is SUMO-1-GCK(2-19)NHNH 23 shows two peaks, both with the mass of the first intermediate in thioesterification (Schiff base) ${ }^{38} \mathbf{4}$ is the final SUMO-GCK(2-19)-MPAA, and $\mathbf{5}$ is the second intermediate in thioesterification (Knorr pyrazole).$^{38} *$ shows a mass that most likely is truncated protein from expression of the intein, which coeluted with all intermediate products. The slow rate of thioesterification is attributed to a C-terminal Ile ${ }^{41} ;$ B. Overlay of the simulated HR-MS (above) and deconvoluted HR-MS (below), and $\mathbf{C}$. HPLC trace for the final product SUMO-GCK(2-19)-MPAA.

\section{Conclusions}

Lysine, often a site of ubiquination, SUMOylation, and other Ubl protein PTM conjugations, was designed with an artificial $\gamma$-selenol moiety. The resulting amino acid was successfully used for traceless isopeptide ligation of SUMO-1 on a peptide of its natural target protein, human glucokinase. Additionally, a selenolysine analog, possessing orthogonal protecting groups to be used in traceless dual chemical ligation, was designed and synthesized. Selenolysine presents an attractive building block in the synthesis of post-translationally modified proteins, especially useful for those containing native Cys residues in their sequences.

\section{Materials/Methods}


All details for synthesis and characterization can be found in the Supporting Information.

\section{Acknowledgements}

We thank the Dr. Tsafi Danieli, Dr. Mario Lebendiker, and Dr. Yael Keren for their assistance in SUMO1-COSR expression and purification in the Wolfson Centre for Applied Structural Biology at HUJI. Thanks to Dr. Bill Breuer of the Mass Spectrometry Unit at the Institute of Life Sciences, HUJI for assistance with high resolution mass spectrometry. We wish to thank Prof. Dr. Huib Ovaa (Leiden University Medical Center, The Netherlands) for the generous gift of chlorolysine. RND was supported by the Kaete Klausner Ph.D. scholarship, SK was supported by PBC fellowship for postdocs, NM acknowledges the support of ISF (783/18) and the US-Israel Binational Science Foundation (BSF) (2014167), and BGM acknowledges the support of the NIH (GM115388).

\section{Conflicts of interest}

The authors declare no competing financial interest.

\section{Bibliography}

1. F. Wold, Annu. Rev. Biochem., 1981, 50, 783-814.

2. X. J. Yang, Oncogene, 2005, 24, 1653-1662.

3. R. L. Welchman, C. Gordon and R. J. Mayer, Nat. Rev. Mol. Cell Biol., 2005, 6, 599-609.

4. P. Siman and A. Brik, Org. Biomol. Chem., 2012, 10, 5684-5697.

5. J. M. Chalker, S. B. Gunnoo, O. Boutureira, S. C. Gerstberger, M. FernándezGonzález, G. J. L. Bernardes, L. Griffin, H. Hailu, C. J. Schofield and B. G. Davis, Chem. Sci., 2011, 2, 1666-1676.

6. D. H. Strumeyer, W. N. White and D. E. Koshland, Jr., Proc. Natl. Acad. Sci. USA, 1963, 50, 931-935.

7. T. J. J. Holmes and R. G. Lawton, J. Am. Chem. Soc., 1977, 99, 1984-1986.

8. G. J. L. Bernardes, J. M. Chalker, J. C. Errey and B. G. Davis, J. Am. Chem. Soc., 2008, 130, 5052-5053.

9. R. Mousa, R. N. Dardashti and N. Metanis, Angew. Chem. Int. Ed., 2017, 56, 15818-15827.

10. R. Meledin, S. M. Mali, S. K. Singh and A. Brik, Org. Biomol. Chem., 2016, 14, 4817-4823.

11. G. G. Kochendoerfer and S. B. H. Kent, Curr. Opin. Chem. Biol., 1999, 3, 665-671.

12. R. B. Merrifield, J Am Chem Soc, 1963, 85, 2149-2154.

13. P. E. Dawson, T. W. Muir, I. Clark-Lewis and S. B. H. Kent, Science, 1994, 266, 776-779. 
14. C. Chatterjee, R. K. McGinty, J. P. Pellois and T. W. Muir, Angew. Chem. Int. Ed., 2007, 46, 2814-2818.

15. R. K. McGinty, J. Kim, C. Chatterjee, R. G. Roeder and T. W. Muir, Nature, 2008, 453, 812-816.

16. K. S. A. Kumar, M. Haj-Yahya, D. Olschewski, H. A. Lashuel and A. Brik, Angew. Chem. Int. Ed., 2009, 48, 8090-8094.

17. H. P. Hemantha, S. N. Bavikar, Y. Herman-Bachinsky, N. Haj-Yahya, S. Bondalapati, A. Ciechanover and A. Brik, J. Am. Chem. Soc., 2014, 136, 26652673.

18. M. Seenaiah, M. Jbara, S. M. Mali and A. Brik, Angew. Chem. Int. Ed., 2015, 54, 12374-12378.

19. R. Yang, K. K. Pasunooti, F. Li, X. W. Liu and C. F. Liu, J. Am. Chem. Soc., 2009, 131, 13592-13593.

20. R. Yang, K. K. Pasunooti, F. Li, X. W. Liu and C. F. Liu, Chem Commun, 2010, 46, 7199-7201.

21. N. Metanis, E. Keinan and P. E. Dawson, Angew. Chem. Int. Ed., 2010, 49, 70497053.

22. S. Dery, P. S. Reddy, L. Dery, R. Mousa, R. Notis Dardashti and N. Metanis, Chem. Sci., 2015, 6, 6207-6212.

23. R. Merkx, G. de Bruin, A. Kruithof, T. van den Bergh, E. Snip, M. Lutz, F. El Oualid and H. Ovaa, Chem. Sci., 2013, 4, 4494-4498.

24. A. H. G. Siebum, W. S. Woo, J. Raap and J. Lugtenburg, Eur. J. Org. Chem. , 2004, DOI: 2905-2913, 2905-2913.

25. J. M. Padron, G. Kokotos, T. Martin, T. Markidis, W. A. Gibbons and V. S. Martin, Tetrahedron-Asymmetr, 1998, 9, 3381-3394.

26. J. M. Padrón, G. Kokotos, T. Martín, T. Markidis, W. A. Gibbons and V. S. Martin, Tetrahedron-Asymmetr, 1998, 9, 3381-3394.

27. M. D. Gieselman, L. Xie and W. A. van der Donk, Org. Lett., 2001, 3, 1331-1334.

28. J. N. Hernández, M. A. Ramírez and V. S. Martin, J Org Chem, 2003, 68, 743-746.

29. C. Castaneda, J. Liu, A. Chaturvedi, U. Nowicka, T. A. Cropp and D. Fushman, J. Am. Chem. Soc., 2011, 133, 17855-17868.

30. I. Aukrust, L. Bjørkhaug, M. Negahdar, J. Molnes, B. B. Johansson, Y. Müller, W. Haas, S. P. Gygi, O. Søvik, T. Flatmark, R. N. Kulkarni and P. R. Njølstad, J. Biol. Chem., 2013, 288, 5951-5962.

31. B. B. Johansson, K. Fjeld, M. H. Solheim, J. Shirakawa, E. Zhang, M. Keindl, J. $\mathrm{Hu}$, A. Lindqvist, A. Døskeland, G. Mellgren, T. Flatmark, P. R. Njølstad, R. N. Kulkarni, N. Wierup, I. Aukrust and L. Bjørkhaug, Mol. Cell. Endocrinol., 2017, 454, 146-157.

32. J. Xu, Y. He, B. Qiang, J. Yuan, X. Peng and X. M. Pan, Bmc Bioinformatics, 2008, 9.

33. J. Ren, X. J. Gao, C. J. Jin, M. Zhu, X. W. Wang, A. Shaw, L. P. Wen, X. B. Yao and Y. Xue, Proteomics, 2009, 9, 3409-3412.

34. K. Kamata, M. Mitsuya, T. Nishimura, J. I. Eiki and Y. Nagata, Structure, 2004, 12, 429-438.

35. M. S. Rodriguez, C. Dargemont and R. T. Hay, J. Biol. Chem., 2001, 276, 1265412659.

36. P. Petit, M. Antoine, G. Ferry, J. A. Boutin, A. Lagarde, L. Gluais, R. Vincentelli and L. Vuillard, Acta Crystallogr D Biol Crystallogr, 2011, 67, 929-935.

37. J. S. Zheng, S. Tang, Y. K. Qi, Z. P. Wang and L. Liu, Nat. Protoc., 2013, 8, 24832495. 
38. D. T. Flood, J. C. J. Hintzen, M. J. Bird, P. A. Cistrone, J. S. Chen and P. E. Dawson, Angew Chem Int Edit, 2018, 57, 11634-11639.

39. J. Bouchenna, M. Senechal, H. Drobecq, J. Vicogne and O. Melnyk, Bioconjugate Chem, 2019, 30, 2967-2973.

40. N. H. Shah, G. P. Dann, M. Vila-Perelló, Z. Liu and T. W. Muir, J. Am. Chem. Soc., 2012, 134, 11338-11341.

41. T. M. Hackeng, J. H. Griffin and P. E. Dawson, Proc. Natl. Acad. Sci. USA, 1999, 96, 10068-10073.

42. P. S. Reddy, S. Dery and N. Metanis, Angew. Chem. Int. Ed., 2016, 55, 992-995.

43. H. Rohde, J. Schmalisch, Z. Harpaz, F. Diezmann and O. Seitz, Chembiochem : a European journal of chemical biology, 2011, 12, 1396-1400.

44. R. N. Dardashti and N. Metanis, Bioorg. Med. Chem., 2017, 25, 4983-4989.

45. J. Bouchenna, M. Senechal, H. Drobecq, N. Stankovic-Valentin, J. Vicogne and O. Melnyk, Bioconjugate Chem, 2019, 30, 2684-2696.

46. J. Molnes, K. Teigen, I. Aukrust, L. Bjørkhaug, O. Søvik, T. Flatmark and P. R. Njølstad, FEBS J., 2011, 278, 2372-2386. 
